\title{
Acute Adrenal Crisis in an Asthmatic Child Treated with Inhaled Fluticasone Proprionate
}

\author{
Angela H. Santiago and Susan Ratzan \\ Division of Pediatric Endocrinology, Connecticut Children's Medical Center, University of Connecticut, Hartford, CT 06106, USA \\ Correspondence should be addressed to Angela H. Santiago, docangela2002@yahoo.com
}

Received 3 March 2010; Revised 30 June 2010; Accepted 13 July 2010

Academic Editor: Deborah Merke

Copyright () 2010 A. H. Santiago and S. Ratzan. This is an open access article distributed under the Creative Commons Attribution License, which permits unrestricted use, distribution, and reproduction in any medium, provided the original work is properly cited.

\begin{abstract}
Adrenal suppression secondary to prolonged inhaled corticosteroid use is usually limited to biochemical abnormalities, with no obvious clinical effects. Acute adrenal crisis is much rarer event but has been reported with increasing frequency. We report a case of a 7-year-old asthmatic child who presented with an acute history of lethargy after a respiratory infection. He was maintained on $220 \mu \mathrm{g}$ /day of fluticasone propionate for several years. Initial evaluation revealed severe adrenal suppression, with undetectable cortisol levels and minimal response after stimulation with ACTH. After fluticasone was discontinued, a gradual recovery of the adrenal axis was seen. This case shows that acute adrenal crisis may be a consequence even at the usual prescribed doses, stressing the importance of using the lowest dose of inhaled steroids needed to control symptoms and having an increased awareness of this complication.
\end{abstract}

\section{Introduction}

Inhaled corticosteroids (ICSs) are a cornerstone for the treatment of asthma, proven to be very effective in its decades of use. They prevent asthma exacerbations, maximize lung function, and are the only treatment for chronic asthma shown to reduce asthma-related deaths [1]. Inhaled steroids include beclomethasone, budesonide, fluticasone dipropionate, mometasone furoate, and triamcinolone acetate. While all inhaled steroids work by binding to a common glucocorticoid receptor, the potency of each medication differs, as well as the side effect profile. Fluticasone propionate has been in use since the 1990s and is more potent than its predecessors. It has equivalent efficacy when given at half the daily dose of the older medications, beclomethasone diproprionate and budesonide [2]. Its favorable pharmacologic properties include a higher affinity for glucocorticosteroid receptors, higher lipophilicity, and greater lung deposition. Side effects were thought to be lower due to less oral systemic absorption due to an almost complete first pass hepatic clearance (almost 99\%) of the swallowed part of the medication (at least $70 \%$ of emitted dose) [3, 4]. At the recommended daily dose in children of $100-200 \mu \mathrm{g}$, there is a good safety profile, with limited effects on the adrenal function or growth. However, such side effects are seen starting at a dose of $400 \mu \mathrm{g} /$ day, particularly when given for a prolonged period [5]. When adrenal suppression is seen in patients, it is usually limited to laboratory abnormalities. Acute adrenal insufficiency is less common but has been reported with increasing frequency over the past decade, especially as fluticasone use has become widespread [4]. We report a case of a 7 -year-old asthmatic child who presented with adrenal crisis while on a dose of $220 \mu \mathrm{g} /$ day of fluticasone proprionate, lower than the usual suppressive dose.

\section{Case}

A 7-year-old asthmatic child presented to the emergency department with a 1 day history of fever, cough, vomiting, and lethargy. He had been maintained on inhaled fluticasone, $220 \mu \mathrm{g} /$ day (110 ug BID) for the past four years. He had briefly used a $440 \mu \mathrm{g} /$ day dose for a few months two years prior. He has no oral prednisone use for the past year. Significant in the past medical history is an episode of acute gastroenteritis 10 months prior, which led to hypovolemic 
shock, accompanied by hypoglycemia and altered mental status. He was admitted to the PICU and required fluid resuscitation and vasopressors. He recovered completely from this. No adrenal work-up was done at that time. In the present ED visit, initial vital signs showed a BP 108/60, HR128/min, RR 24/min, temperature 101.2 F. He appeared lethargic and acutely ill. The rest of the physical exam was unremarkable. Initial lab work revealed normal electrolytes and liver function, with a normal glucose of $116 \mathrm{mg} / \mathrm{dl}$ $(6.4 \mathrm{mmol} / \mathrm{L})$. He had a normal WBC, but with granulocyte predominance. Cortisol at that time was $<8.28 \mathrm{nmol} / \mathrm{L}$ (normal afternoon cortisol $63.48-328.44 \mathrm{nmol} / \mathrm{L}$ ). He was given intravenous normal saline and IV hydrocortisone, which provided immediate improvement. Consultation with a pediatric endocrinologist was made, who noted that he had a mildly Cushingoid appearance. There was no skin hyperpigmentation. A review of the growth chart showed a decline of his height from the $60 \%$ to the $13 \%$ on the preceding year while his weight increased 55\% from $65 \%$. A $250 \mu \mathrm{g}$ ACTH stimulation test was done, where a basal cortisol of $19.32 \mathrm{nmol} / \mathrm{L}$ only increased to $35.88 \mathrm{nmol} / \mathrm{L}$ in 60 mins. Because of the history of receiving inhaled steroids, secondary adrenal suppression was suspected. Prolonged stimulation test was performed (8-hour ACTH infusion for 3 days), with cortisol level peaking to $361.56 \mathrm{nmol} / \mathrm{L}$ on day 3. This showed an ability of the adrenals to respond, making secondary adrenal suppression more likely. The ACTH level drawn in the ED came back at this time and was elevated at $89.98 \mathrm{pmol} / \mathrm{L}$ (normal $1.3-10.56 \mathrm{pmol} / \mathrm{L}$ ). Adrenal antibodies were negative. With the guidance of a pulmonologist, the child was completely weaned off inhaled steroids and switched to oral Singulair (Montelukast). The child recovered quickly with no complications. The family was taught to do stress dosing with hydrocortisone in case of illness. On outpatient followup a month after steroid discontinuation, he remained well. Electrolytes remained normal, morning (AM) cortisol was $110.4 \mathrm{nmol} / \mathrm{L}$ (normal $171.12-535.4 \mathrm{nmol} / \mathrm{L}$ ), and ACTH level was $22.6 \mathrm{pmol} / \mathrm{L}$ (see Table 1). Two months off steroids, AM cortisol was $187.68 \mathrm{nmol} / \mathrm{L}$ and ACTH level was $22.2 \mathrm{pmol} / \mathrm{L} .9$ months off steroids, his AM cortisol was $292.56 \mathrm{nmol} / \mathrm{L}$ and ACTH was $14.74 \mathrm{pmol} / \mathrm{L} .16$ months after, a low-dose $(1 \mu \mathrm{g})$ ACTH stimulation test showed a peak cortisol of $477.48 \mathrm{nmol} / \mathrm{L}$. Throughout this period, the child needed stress dosing once for a viral illness from which he recovered immediately. His asthma has remained well controlled on his current regimen. He had catch-up growth, with height now at the 35th percentile, and his Cushingoid appearance has resolved.

\section{Discussion}

Secondary adrenal suppression is a well-known complication of long-term administration of high doses of steroids, with different propensities depending on the route of administration. Excess steroids result in negative feedback on the hypothalamic-pituitary-adrenal axis, rendering the body incapable of mounting a stress response in times of increased need. Symptoms of adrenal insufficiency may be nonspecific like fatigue, anorexia, vomiting, and abdominal pain but may also lead to a life-threatening adrenal crisis accompanied by shock $[5,6]$. Inhaled corticosteroids (ICSs) have been widely used since its introduction, not only because of its efficacy, but also because it is thought to have decreased toxicity from the lower systemic absorption compared to oral steroids. However, toxicity is commonly seen especially with prolonged use of high doses.

Multiple studies have attempted to estimate the propensity of inhaled corticosteroids (ICSs) to cause adrenal suppression. In a systematic review of literature from 1973 to 2005,22 studies were analyzed to evaluate hypothalamicpituitary-adrenal axis suppression from inhaled steroids, as determined by gold standard tests like metyrapone or insulin tolerance tests. The best data was a cohort study published in 1982 by Vaz et al. showing that there was a $100 \%$ absolute risk of adrenal suppression in asthmatic children treated with beclomethasone metered dose inhaler at a dose of $250-600 \mu \mathrm{g} / \mathrm{m}^{2} /$ day for 6-42 months. Studies suggest that this may be similar with other ICSs $[7,8]$. A meta-analysis examined dose-related response and adrenal suppression of inhaled fluticasone, assessing double blinded randomized controlled trials from 1980 to 2002 and included seven studies of 1733 children. Based on a dose response curve, fluticasone's efficacy seems to plateau between 100 and $200 \mu \mathrm{g}$. A dose of $400 \mu \mathrm{g}$ was shown to give additional benefit particularly for severe asthmatics, but adrenal suppression also was seen at this dose [9]. In a study of 220 children on high dose ( $>500 \mu \mathrm{g} /$ day $)$ inhaled fluticasone, a low-dose ACTH test showed that $45 \%$ of the children had flat or impaired response (peak cortisol $<500 \mathrm{nmol} / \mathrm{L}$ ) [10]. While biochemical adrenal suppression is well described, acute adrenal crisis is less common, and the actual prevalence is not known. In a review of inhaled corticosteroid use in 1994, Russell wrote that there was no firm evidence of adrenal crisis [11]. Until 1999, there were only 2 cases of adrenal crises reported in its 30 years of use. Since then, there have been an increasing number of reports of adrenal crisis, including case reports, case series, and a survey [12]. In the survey done in UK, 33 patients (28 children, 5 adults) were identified to have adrenal crisis. 23 of the children presented with hypoglycemia, accompanied with decreased level of consciousness, seizures, or death in 1 . All identified patients were treated with 500-2000 $\mu \mathrm{g}$ /day ICS, with $94 \%$ receiving fluticasone. The majority $(76 \%)$ of the patients were treated for at least a year. Fluticasone accounted for the smallest percentage of prescriptions for ICS in that area, making the authors conclude that fluticasone had a higher propensity for adrenal suppression [4]. A possible explanation for this is fluticasone's high lipophilicity. While the high hepatic clearance of fluticasone offers less systemic absorption, absorption via the lungs into the bloodstream remains an important source of systemic ICS, as this escapes first pass metabolism. Fluticasone is $200-300$ times more lipophilic than budesonide and beclomethasone and results in a greater volume of distribution. This causes higher binding in peripheral tissues, longer elimination half-life, and more accumulation [4]. 
TABLE 1: Patient's laboratory results showing initial adrenal suppression and subsequent recovery after steroid discontinuation.

\begin{tabular}{|c|c|c|c|c|}
\hline & \multicolumn{2}{|c|}{ Cortisol (nmol/L) } & \multicolumn{2}{|c|}{$\mathrm{ACTH}(\mathrm{pmol} / \mathrm{L})$} \\
\hline & Patient values & Normal range & Patient values & Normal range \\
\hline \multicolumn{5}{|l|}{ (a) On inhaled steroids } \\
\hline Initial & $<8.3$ & 63.5-328.4 (PM) & 409 & $1.3-10.56$ \\
\hline $\begin{array}{l}250 \mu \mathrm{g} \text { ACTH test, } \\
\text { Baseline }\end{array}$ & 19.3 & & & \\
\hline $\begin{array}{l}250 \mu \mathrm{g} \text { ACTH test, } \\
\text { Stimulated }\end{array}$ & 35.9 & $>500$ & & \\
\hline 3 day ACTH-peak & 361.6 & $>500$ & & \\
\hline \multicolumn{5}{|c|}{ (b) After steroid discontinuation } \\
\hline 1 month & 110.4 & $171.1-535.4(\mathrm{AM})$ & 22.6 & $1.3-10.56$ \\
\hline 2 months & 187.68 & $171.1-535.4$ & 22.2 & \\
\hline 9 months & 292.56 & $171.1-535.4$ & 14.7 & \\
\hline $\begin{array}{l}16 \text { months } \\
(1 \mu \mathrm{g} \text { ACTH test, peak) }\end{array}$ & 477.48 & $>500$ & 7.04 & \\
\hline
\end{tabular}

Aside from differences between the inhaled steroids, interindividual variations may also account for differences in glucocorticoid sensitivity. This may be due to differences in cortisol metabolism, as well as genetic variations in the glucocorticoid receptor (GR). Steroids act primarily through a target cytoplasmic GR, which is part of a more complicated GR complex with various components. Due to the complexity of this complex, there has been so far limited pharmacogenetic information useful in the clinical setting. There have been mutations identified with NR3C1, which is the gene encoding for the GR, mostly associated with glucocorticoid resistance [13]. Variations in codon 363 of the glucocorticoid receptor (N363S) have been associated with an increased sensitivity to glucocorticoids in vivo [14]. Drug-drug interactions also may account for increased toxicity from inhaled steroids. Fluticasone as well as budesonide is metabolized mainly by CYP3A4, and drugs that inhibit this enzyme increase the bioavailability of the inhaled steroids, just as in steroids taken orally and parenterally. This subsequently increases the risk for systemic adverse reactions. This is well demonstrated in a case series of 46 patients on inhaled steroids who developed adrenal insufficiency. Fifteen of the cases were possibly due to a drug interaction with known cytochrome p450 inhibitors, including itraconazole, ritonavir, verapamil, and diltiazem [15]. Other known inhibitors include etomidate, diltiazem, and grapefruit juice. Drugs that activate cortisol metabolism may also cause adrenal insufficiency in patients with limited adrenal reserves, or those on replacement glucocorticoid treatment. This includes phenytoin, barbiturates, and rifampin [5]. The child in our case report had no history of use of any of the aforementioned medications.

There are several etiologies which must be considered for a childhood onset adrenal disease. Hereditary disorders include autoimmune adrenal disease, which may be part of autoimmune polyglandular syndrome. In the Western hemisphere, this autoimmune disease is responsible for up to $80 \%$ of cases of primary adrenal failure [16]. Our patient had negative antiadrenal antibodies on screening.
Adrenomyeloneuropathy is a milder form of the $\mathrm{x}$-linked recessive disorder adrenoleukodystrophy, both occurring at frequency of 1 in 20,000 males, and may account for up to $10 \%$ of all cases of adrenal failure. Both present with adrenal insufficiency as well as spasticity and weakness. Adrenomyeloneuropathy has a later onset at adolescence and adulthood and has a slower and milder course. A vital consideration is that adrenal insufficiency may be the only presenting symptom in this condition. The defect is in the beta-oxidation of fatty acids in the peroxisomes, and diagnosis is shown by elevated serum very-longchain fatty acids (VLCFA) [16]. Other etiologies include late onset congenital adrenal hyperplasia (such as from 21-hydroxylase deficiency), congenital adrenal hypoplasia, infiltrative adrenal disease (tuberculosis, HIV, and fungal infection), and adrenal hemorrhage. With the absence of other associated clinical signs and symptoms, and adrenal recovery after steroid weaning in this patient, a complete work-up for these other etiologies was not pursued.

The child's initial blood tests of low cortisol and high ACTH levels, as well as the poor response to the standard ACTH stimulation test, can be suggestive of primary adrenal disease. However, the child did not have any signs of aldosterone deficiency (he had normal electrolytes) and no clinical signs of skin hyperpigmentation and salt craving which usually accompany a primary adrenal problem. There remained a strong suspicion of secondary adrenal suppression from his clinical history, so a prolonged ACTH test was then done. In most cases, this test is rarely necessary as clinical history, physical exam, and single-dose ACTH testing may provide the diagnosis. However, for certain patients like ours where the diagnosis may not be as clear cut, this can help further differentiate primary from secondary/tertiary cause of adrenal insufficiency. $250 \mu \mathrm{g}$ of ACTH was given for 3 days via an 8-hour infusion, with multiple cortisol levels drawn at different time points $[5,17]$. Elevation of plasma cortisol level in the child at the last day of the infusion confirmed the secretory capacity of the adrenals. The gradual recovery of the adrenal axis after steroid weaning further strengthened 
the diagnosis of secondary adrenal suppression. The initial blood tests with an elevated ACTH level can be explained by the quicker recovery of the hypothalamic-pituitary axis from secondary suppression as compared to the adrenal gland.

This case provides further evidence that potentially life-threatening adrenal crisis may occur from prolonged inhaled corticosteroid use. Package insert for fluticasone (in particular Flovent) recommends $88 \mu \mathrm{g}$ BID for children less than 12 years old, but many physicians use it at higher doses particularly in severe asthma. While most cases of adrenal crisis are seen with $>400 \mu \mathrm{g} /$ day, this child was maintained on $220 \mu \mathrm{g} /$ day for the past year, stressing the role of individual patient sensitivity to steroids. The child's history is significant for hypovolemic shock following an acute gastroenteritis, which in hindsight was likely to be an episode of adrenal crisis. Unfortunately, suspicion of adrenal insufficiency arose only on the more recent ED visit. This emphasizes the importance for physicians to be aware of this complication, especially as this is a very commonly prescribed medication. It is therefore vital to periodically reassess a child's asthma status, with the aim of tapering to the lowest effective dose and subsequently minimizing systemic side effects. For evaluation of at-risk patients, the low-dose $(0.5-1 \mu \mathrm{g})$ ACTH stimulation test is thought to be more sensitive for diagnosing secondary adrenal suppression, as the usual $250 \mu \mathrm{g}$ dose is supraphysiologic and might lead to false negative results. However, some studies do not support the superiority of one test over the other, particularly as both may lead to false negative results [15].

\section{Conclusion}

Inhaled steroids are indispensible in the treatment of chronic asthma, but their prolonged use at doses higher than recommended increases the risk of adrenal suppression. This case shows that life-threatening adrenal crisis may be a consequence even at the usual prescribed doses, stressing the importance of using the lowest dose of inhaled steroids needed to control symptoms and being vigilant of side effects.

\section{References}

[1] N. C. Barnes, "The properties of inhaled corticosteroids: similarities and differences," Primary Care Respiratory Journal, vol. 16, no. 3, pp. 149-154, 2007.

[2] N. Adams, T. J. Lasserson, C. J. Cates, and P. W. Jones, "Fluticasone versus beclomethasone or budesonide for chronic asthma in adults and children," Cochrane Database of Systematic Reviews, no. 2, Article ID CD002310, 2005.

[3] V. Marchac, V. Foussier, P. Devillier, M. Le Bourgeois, and M. Polak, "Fluticasone propionate in children and infants with asthma," Archives de Pediatrie, vol. 14, no. 4, pp. 376-387, 2007.

[4] G. R. G. Todd, C. L. Acerini, R. Ross-Russell, S. Zahra, J. T. Warner, and D. McCance, "Survey of adrenal crisis associated with inhaled corticosteroids in the United Kingdom," Archives of Disease in Childhood, vol. 87, no. 6, pp. 457-461, 2002.
[5] E. Charmandari and G. Chrousos, "Adrenal insufficiency," in Endotext: Adrenal Disease and Function, chapter 13, Endotext.com, 2003.

[6] G. Reimondo, S. Bovio, B. Allasino, M. Terzolo, and A. Angeli, "Secondary hypoadrenalism," Pituitary, vol. 11, no. 2, pp. 147154, 2008.

[7] M. Masoli, M. Weatherall, S. Holt, and R. Beasley, "Systematic review of the dose-response relation of inhaled fluticasone propionate," Archives of Disease in Childhood, vol. 89, no. 10, pp. 902-907, 2004.

[8] R. Vaz, B. Senior, M. Morris, and A. Binkiewicz, "Adrenal effects of beclomethasone inhalation therapy in asthmatic children," Journal of Pediatrics, vol. 100, no. 4, pp. 660-662, 1982.

[9] E. W. Zöllner, "Hypothalamic-pituitary-adrenal axis suppression in asthmatic children on inhaled corticosteroids (part 2) - the risk as determined by gold standard adrenal function tests: a systematic review," Pediatric Allergy and Immunology, vol. 18, no. 6, pp. 469-474, 2007.

[10] J. Paton, E. Jardine, E. McNeill et al., "Adrenal responses to low dose synthetic ACTH (Synacthen) in children receiving high dose inhaled fluticasone," Archives of Disease in Childhood, vol. 91, no. 10, pp. 808-813, 2006.

[11] G. Russell, "Inhaled corticosteroid therapy in children: an assessment of the potential for side effects," Thorax, vol. 49, no. 12, pp. 1185-1188, 1994.

[12] A. J. Drake, R. J. Howells, J. P. H. Shield, A. Prendiville, P. S. Ward, and E. C. Crowne, "Symptomatic adrenal insufficiency presenting with hypoglycaemia in asthmatic children with asthma receiving high dose inhaled fluticasone propionate," British Medical Journal, vol. 324, no. 7345, pp. 1081-1082, 2002.

[13] G. A. Hawkins and S. P. Peters, "Pharmacogenetics of asthma," Methods in Molecular Biology, vol. 448, pp. 359-378, 2008.

[14] N. A. T. M. Huizenga, J. W. Koper, P. De Lange et al., "A polymorphism in the glucocorticoid receptor gene may be associated with an increased sensitivity to glucocorticoids in vivo," Journal of Clinical Endocrinology and Metabolism, vol. 83, no. 1, pp. 144-151, 1998.

[15] A. Daveluy, C. Raignoux, G. Miremont-Salamé et al., "Drug interactions between inhaled corticosteroids and enzymatic inhibitors," European Journal of Clinical Pharmacology, vol. 65, no. 7, pp. 743-745, 2009.

[16] S. R. Bornstein, "Predisposing factors for adrenal insufficiency," The New England Journal of Medicine, vol. 360, no. 22, pp. 2328-2339, 2009.

[17] L. I. Rose, G. H. Williams, P. I. Jagger, and D. P. Lauler, "The 48-hour adrenocorticotrophin infusion test for adrenocortical insufficiency," Annals of Internal Medicine, vol. 73, no. 1, pp. 49-54, 1970. 